\title{
The characteristics analysis of strain variation associated with Wenchuan earthquake using principal component analysis
}

\author{
Kaiguang Zhu*, Chengquan Chi, Zining Yu, Mengxuan Fan, Kaiyan Li, Huihui Sun \\ Key Laboratory of Geo-Exploration Instrumentation, Ministry of Education, Jilin University, Changchun, China
}

Article history: received September 28, 2018; accepted September 24, 2019

\begin{abstract}
Borehole strainmeters that are installed deeply into bedrock are capable of recording both continuous stress and strain measurements, and have consequently become an important tool for monitoring crustal deformation. A YRY-4 borehole strainmeter installed at the Guza Station recorded anomalous changes in borehole strain data preceding the Wenchuan earthquake on May 12, 2008 (UTC) (=8.0). We apply principal component analysis (PCA) to analyze borehole strain data from the Guza Station. The first principal component eigenvalues and eigenvectors are calculated. The fitted results of the cumulative number of anomalous eigenvalues demonstrate that an acceleration occurred approximately 4 months before the earthquake (from January 2008). The results of the combined eigenvalue and eigenvector analyses show that the spatial distribution of eigenvectors and accelerated occurrence of eigenvalue anomalies represents the stress evolution characteristics of the fault from a steady state to a sub-instability state in rock experiments. We tentatively infer that this process may also be linked to the preparation phase of a large earthquake.
\end{abstract}

Keywords: Borehole strain; the Wenchuan earthquake; principal component analysis; eigenvalue and eigenvector; earthquake precursor.

\section{Introduction}

The first borehole dilatometer was invented by Sacks, of Carnegie Institution of Washington, and Evertson, of University of Texas, in September of 1968 [Sacks and Evertson, 1971]. Since then, the multi-component borehole strainmeters have appeared in succession, including the RZB-1 four-gauge borehole strainmeter [Ouyang, 1977], the Gladwin three-component borehole strainmeter [Gladwin, 1984], and so on. Thanks to the Plate Boundary Observatory (PBO) project, the development of the high-resolution borehole strainmeters in China has been accelerated [Qiu and Zhang, 2002]. The YRY-4 four-gauge borehole strainmeter, designed by Chi Shunliang, has been developed and there are more than 40 four-gauge borehole strainmeters sites in China [Chi et al., 2009]. Earthquakes will deform the Earth's crust and borehole strain observation play an important role in studying this crustal deformation and the stress field. The observations have many advantages such as high resolution, broad band, and topographic-feature-independence, amongst others. High-resolution borehole strainmeters allow for the detection 


\section{Kaiguang Zhu et al.}

of subtle strain variations to study slow earthquakes [Sacks et al., 1978, 1981; Linde et al.,1996; Liu et al., 2009], seismic strain steps [McGarr et al.,1982; Asai et al.,2005; Qiu et al., 2003, 2004, 2015; Barbour et al., 2012], and volcanic eruptions [Voight et al., 2006; Hautmann et al., 2013; Sturkell et al., 2013]. 1High-resolution borehole records also provide the opportunity to investigate strain changes prior to earthquakes.

The Wenchuan earthquake is the greatest earthquake disaster in China since the 1976 Tangshan earthquake, which occurred in the Longmenshan thrust zone on May 12, 2008 [Fu et al., 2011]. Researchers have studied the precursory phenomena of the Wenchuan earthquake. Chun-Chieh Hsiao et al., [2010] studied the ionospheric electron density variation, and found an abnormal phenomenon around noon within 5 days prior to the Wenchuan earthquake. Liu et al., [2011] used the S transform method to observe that abnormal strain signals increased before the Wenchuan earthquake and decreased after the earthquake. By means of Wavelet Decomposition and Overrun Rate Analysis, [Qiu and Chi et al., 2011] concluded that the abnormal changes observed at the Guza Station (several months before the earthquake) could be related to the Wenchuan earthquake and the anomalous signals do not correspond to seasonal changes.

Principal component analysis (PCA) is a statistical method that is widely employed to reveal relevant information in confusing datasets [Gómez et al., 2005]. It is a non-parametric method that does not utilize deviations from previously described strain changes to determine data anomalies and has the potential to describe the spatial distribution of earthquake-related strain changes. Telesca et al., [2004] discovered earthquake precursor patterns in the daily variations of the principal components of eigenvalues by using PCA to investigate Ultra-Low Frequency (ULF) geoelectrical data. Hattori et al., [2004] applied PCA to the ULF horizontal NS component and indicated that features of eigenvalues and eigenvectors are likel1y to be correlated with large earthquakes. Lin, [2015] examined ionospheric total electron content (TEC) during the time period of the Tohoku earthquake by using two-dimensional principal component analysis, and detected two larger principal eigenvalues on March 11, 2011. In the present work, we have applied PCA to time-series borehole strain data that were recorded at the Guza Station. We aim to detect anomalies in borehole strain that preceded the Wenchuan earthquake and to analyse the cause of these anomalies.

\section{Observations}

The Guza Station is located at the southwestern extent of the Longmenshan fault zone. At the Guza station, the deformation observation instrumentation includes a very-long-period vertical pendulum tiltmeter, YRY-4 borehole strainmeter, DSQ water pipe inclinometer, SS-Y body piercing extensometer, and DZW digitalized gravity meter. The YRY-4 borehole strainmeter was installed in October 2006. Continuous recordings have been collected from this strainmeter at a sampling rate of one sample per minute since December 1, 2006. A seasonal change rule can be seen clearly in the strain data. Therefore, borehole strain data from the Guza Station have a normal background [Qiu et al.,2011].

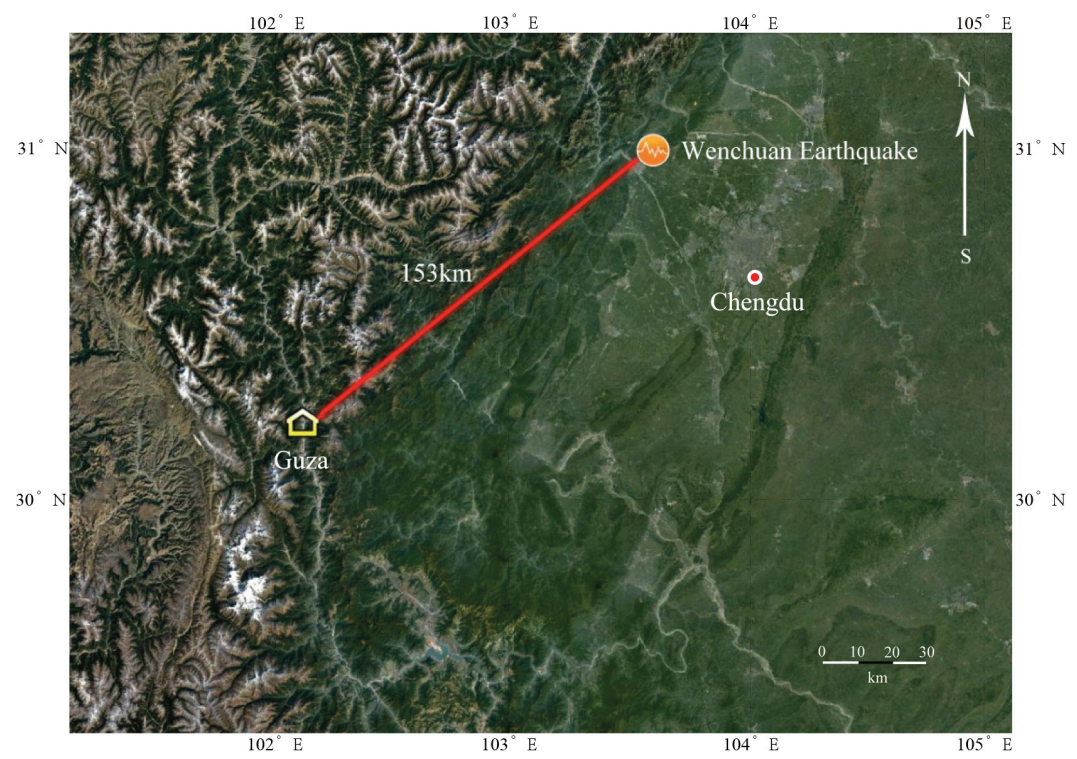

Figure 1. A location map showing the Guza Station and the Wenchuan earthquake epicenter. 
At 14:28(UTC+8) on May 12, 2008, a Ms8.0 earthquake occurred in Wenchuan County, Sichuan. The epicenter was located at $31.01^{\circ} \mathrm{N}$ and $103.42^{\circ} \mathrm{E}$. According to the data published by the China Earthquake Networks Center of the China Earthquake Administration, and the focal depth was approximately $14 \mathrm{~km}$. The Wenchuan earthquake is characterized mainly by thrust motion with right-lateral-strike slip [Fu et al., 2011].

The distance between the epicenter of the Wenchuan earthquake and the receiver at Guza Station is $153 \mathrm{~km}$ (Figure 1). The Wenchuan earthquake had a wide range of influences, and the physical phenomena taking place during earthquake preparation is very complicated. Consequently, we cannot exclude a-priori the possibility that the YRY-4 borehole strainmeter installed at the Guza Station recorded the strain anomaly phenomena taking place during earthquake preparation.

\section{Methodology}

\subsection{Strain conversion}

The YRY-4 borehole strainmeter contains four horizontally emplaced sensors to measure changes in the borehole diameter. The theoretical model is shown in Figure 2.



Figure 2. Sketch of the two-ring system for measuring strain in boreholes.

Gauge $i$ in the cylinder directly measures the change in diameter in the corresponding azimuth $\beta_{i}$ that results from changes in strain state. Although the solutions are complex, the resulting formula of the relationship between the measurement $S_{i}$ and the strain changes $\left(\varepsilon_{1}, \varepsilon_{2}, \alpha\right)$ is straight forward:

$$
S_{i}=A\left(\varepsilon_{1}+\varepsilon_{2}\right)+B\left(\varepsilon_{1}-\varepsilon_{2}\right) \cos 2\left(\beta_{i}-\alpha\right)
$$

The YRY-4 borehole strainmeter contains four horizontally emplaced sensors to measure changes in the borehole diameter. Self-consistency is crucial in FGBS design. According to the theoretical model (1), because the four gauges are arranged at $45^{\circ}$ intervals, the relationship can be obtained as follow:

$$
\left\{\begin{array}{l}
S_{1}=S_{\beta_{1}}=A\left(\varepsilon_{1}+\varepsilon_{2}\right)+B\left(\varepsilon_{1}-\varepsilon_{2}\right) \cos 2\left(\beta_{i}-\mathrm{b} \alpha\right) \\
S_{2}=S_{\beta_{1}+\pi / 4}=A\left(\varepsilon_{1}+\varepsilon_{2}\right)-B\left(\varepsilon_{1}-\varepsilon_{2}\right) \sin 2\left(\beta_{i}-\alpha\right) \\
S_{3}=S_{\beta_{1}+\pi / 2}=A\left(\varepsilon_{1}+\varepsilon_{2}\right)-B\left(\varepsilon_{1}-\varepsilon_{2}\right) \cos 2\left(\beta_{i}-\alpha\right) \\
S_{4}=S_{\beta_{1}+3 \pi / 4}=A\left(\varepsilon_{1}+\varepsilon_{2}\right)+B\left(\varepsilon_{1}-\varepsilon_{2}\right) \sin 2\left(\beta_{i}-\alpha\right)
\end{array}\right.
$$




\section{Kaiguang Zhu et al.}

where $S_{i}(i=1,2,3,4)$ is the measurement obtained from each of the four-gauges. With one additional measurement, a simple relationship among the four measurements can be obtained straight forwardly using equation (2):

$$
S_{1}+S_{2}+S_{3}+S_{4}
$$

which is the self-consistency equation of the YRY-4 borehole strainmeter. This equation can be employed to estimate the credibility of the data. In plane strain problems, all the non-vanishing strains are in a plane while all the out- of-plane components are zero identically in the problem, and borehole strainmeter can only observe two-dimensional strain changes. Hence, there are only three independent variables under plain strain conditions at or near the Earth's surface [Wu et al., 2017]. We can therefore derive various strains from the Guza recordings. The formulas used are as follows:

$$
\left\{\begin{array}{c}
S_{13}=S_{1}-S_{3} \\
S_{24}=S_{2}-S_{4} \\
S_{a}=\left(S_{1}+S_{2}+S_{3}+S_{4}\right) / 2
\end{array}\right.
$$

where $S_{a}$ represents the areal strain, and $S_{13}$ and $S_{24}$ represent the two independent shear strains [Qiu et al., 2013].

\subsection{Principal component analysis}

PCA is a widely used technique in data analysis. It is computationally inexpensive, it can be applied to ordered and unordered attributes, and it can handle both sparse data and skew data. PCA is a non-parametric method that is capable of extracting relevant information from complex data sets [Gómez et al., 2005], and it often reveals relationships that were not previously suspected, thereby allowing for an otherwise unordinary interpretation.

Mathematically, the data are presented in a matrix $\boldsymbol{Y}$ of $m$ rows and $n$ columns:

$$
\boldsymbol{Y}=\left[\begin{array}{ccc}
x_{11} & \cdots & x_{1 n} \\
\vdots & \ddots & \vdots \\
x_{m 1} & \cdots & x_{m n}
\end{array}\right]
$$

where $m$ is the number of samples, and $n$ is the dimension of the sample.

First, we calculate the co-variance matrix $\boldsymbol{C}_{\boldsymbol{Y}}(m \times m)$ of the dataset $\mathbf{Y}(m \times n)$, and the element $\gamma_{p q}$ in the co-variance matrix $\boldsymbol{C}_{\boldsymbol{Y}}(m \times m)$ can be calculated using the following formula:

$$
\gamma_{p q}=[1 /(\mathrm{N}-1)] \sum_{i=1}^{N}\left(x_{p}^{i}-\bar{X}_{p}\right)\left(x_{q}^{i}-\bar{X}_{q}\right)
$$

where $x_{p}^{i}$ and $x_{q}^{i}$ are the $p$ th and $q$ th columns of the $i$ th row of data, respectively, and $\bar{X}_{p}$ and $\bar{X}_{q}$ are the averages of the $p$ th and $q$ th columns of data, respectively. Here $\mathrm{N}$ is the number of samples.

We apply the eigenvalue decomposition to the co-variance matrix:

$$
\boldsymbol{C}_{\boldsymbol{Y}=\boldsymbol{V} \Lambda \boldsymbol{V}^{T}}
$$

where $\Lambda$ is the eigenvalue matrix with $\lambda_{1}, \lambda_{2} \ldots \lambda_{m}\left(\lambda_{1}>\lambda_{2}>\ldots>\lambda_{m}\right)$ and $\boldsymbol{V}$ is the eigenvector matrix whose columns are $\boldsymbol{v}_{1}, \boldsymbol{v}_{\mathbf{2}}, \ldots, \boldsymbol{v}_{\boldsymbol{m}}$. The first principal component eigenvalue and eigenvector are $\lambda_{1}$ and $\boldsymbol{v}_{\mathbf{1}}$, respectively, which represent the principal characteristics of the signals, and other eigenvalues represent information about influencing factors and noise. In this paper, the variations in the eigenvalue and eigenvector of the first principal component are investigated.

We applied PCA to borehole strain data after the strain conversion $\left(S_{13}, S_{24}, S_{a}\right)$, and the co-variance matrix $\boldsymbol{C}_{\boldsymbol{Y}}$ is three dimensions, the obtained eigenvector has three dimensions in the vector space, and it is a unit vector. To present the changes in the eigenvector more intuitively, we transform the eigenvectors to the unit spherical coordinate system, as shown in Figure 3. The eigenvector can be represented by $\theta$ and $\phi$ exclusively. 


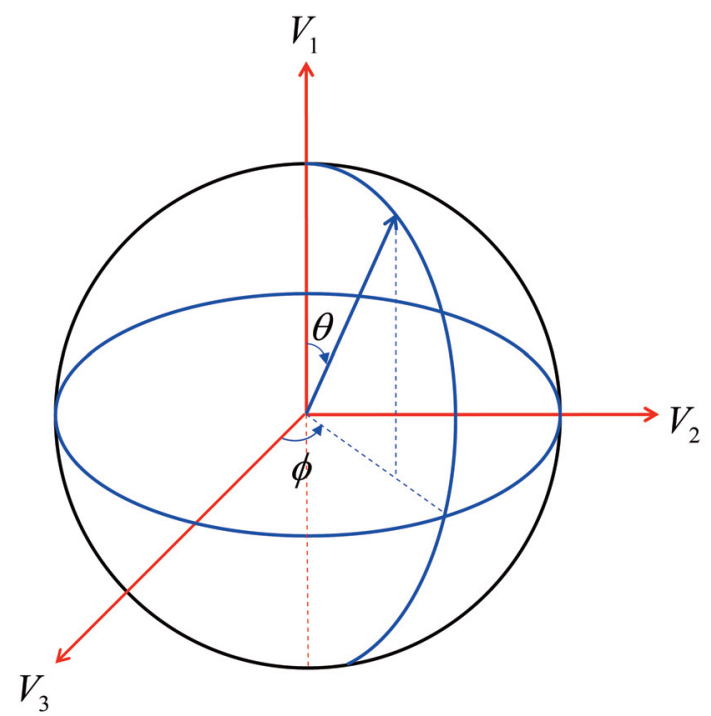

Figure 3. The unit spherical coordinate system of eigenvectors.

\subsection{Data processing}

PCA is applied to extract the anomalies of strain changes associated with the earthquake. We analyze the borehole strain data from the Guza Station that were collected from May 01, 2007, to December 31, 2009. Figure 4 shows the four-component series of borehole strain data that were recorded the Guza Station. At 19:10 on September 12, 2007, a Ms7.9 earthquake occurred in Indonesia. The co-seismic strain changes of the Indonesia earthquake are also recorded by the borehole strainmeter at the Guza station.

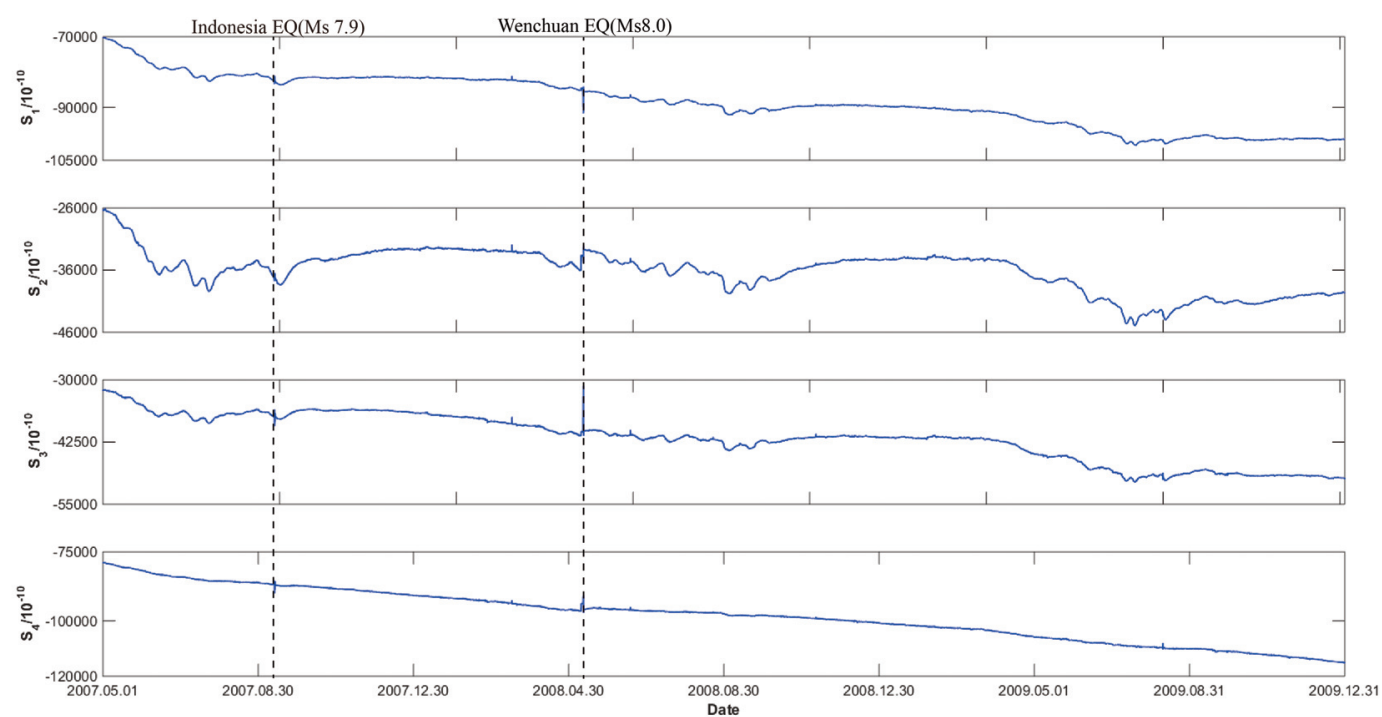

Figure 4. The four-component time series of borehole strain data recorded at the Guza Station, China, from May 01, 2007, to December 31, 2009.

First, the borehole strain data from the Guza Station are checked using the self-consistency equation, after which the four-component borehole strain data are transformed into three components, namely, $S_{13}, S_{24}$, and $S_{a}$, through a strain conversion. A time-series plot of the borehole strain data after the strain conversion is shown in Figure 5. The next step is data preprocessing, in which we remove the influences of solid tide (for which the periods are 24 , 


\section{Kaiguang Zhu et al.}

12,8 , and 6 hours) and the trend reflecting seasonal variation (for which the period is one year) by using a harmoni analysis. The strain changes that reflect the movements of the crust that are associated with the earthquake are regarded as the short period high frequency oscillation signal. A time-series plot of the borehole strain data after data preprocessing are shown in Figure 6. The information regarding the strain change that is related to crustal activity is the most dominant signal in the daily variation data.

The environment surrounding the station is one of the factors affecting borehole strain data. Most of these factors have a cycle of one day. To avoid time-domain aliasing, and to distinguish the anomalous days more easily, we chose to perform PCA on daily data. Let us consider that the three-component data (i.e., 1 day is equal to1440 points) are arranged in the form of time series data vectors.

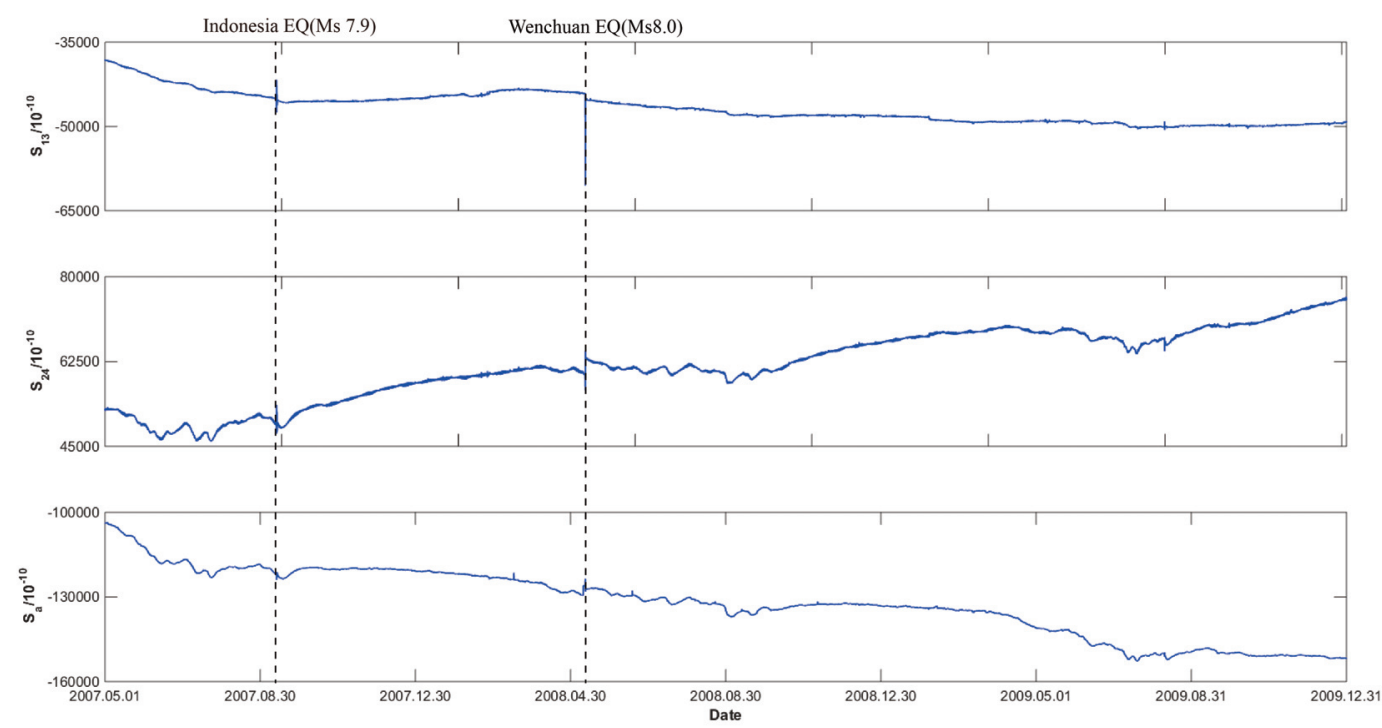

Figure 5. A time series plot of borehole strain data after the strain conversion. $S_{13}$ and $S_{24}$ represent the two independent shear strains, and $\mathrm{S}_{\mathrm{a}}$ represents the areal strain.

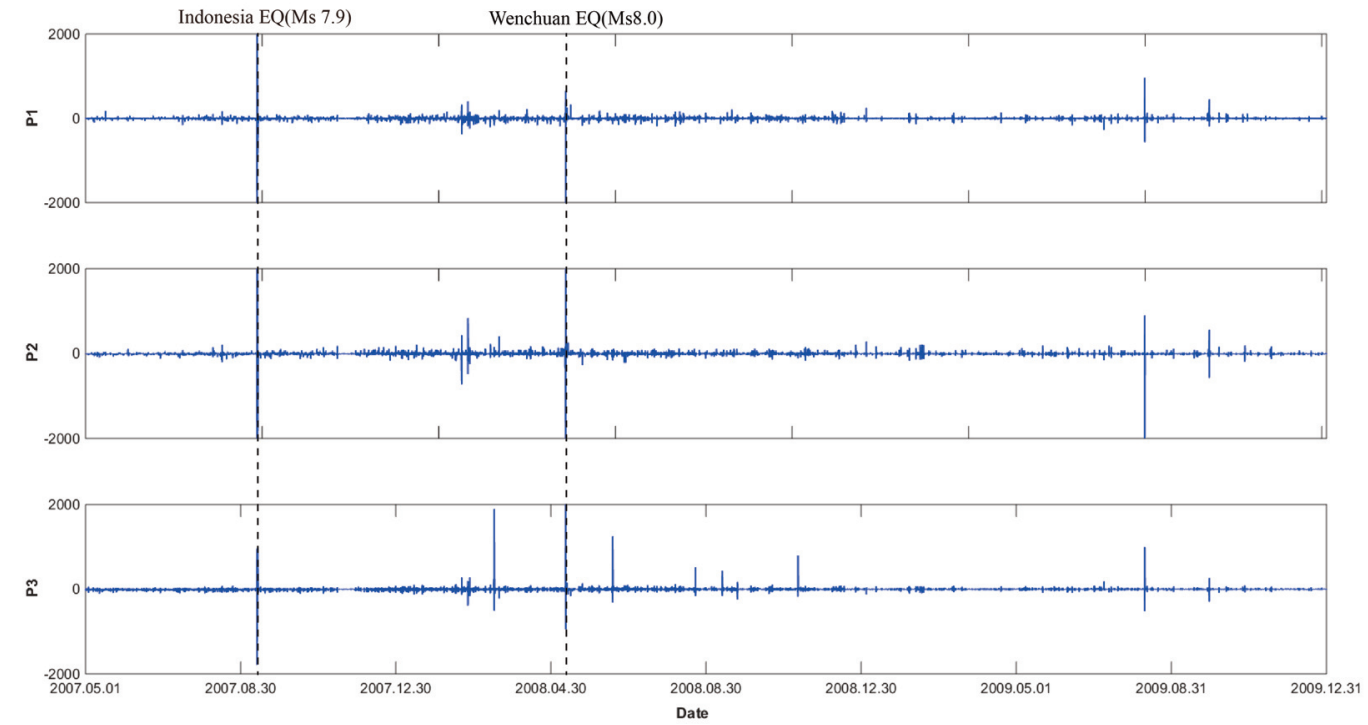

Figure 6. A time series plot of data after data preprocessing. P1, P2 and P3 represent the data after data preprocessing of $\mathrm{S}_{13}, \mathrm{~S}_{24}$ and $\mathrm{S}_{\mathrm{a}}$, respectively. 
Then, we applied PCA to P1, P2 and P3. In the PCA approach, the eigenvector $\boldsymbol{v}_{\mathbf{1}}$ is chosen to maximize the variance in the data. We consider that the eigenvector $\boldsymbol{v}_{\mathbf{1}}$ is the most intense signal subspace [Hattori et al., 2004)] Since we previously transformed the data into three components, it is possible to perform an orthogonal expansion of the signal space into $\boldsymbol{v}_{\mathbf{2}}$ and $\boldsymbol{v}_{\mathbf{3}}$ which are the second and third principal components, respectively.

\section{Results and discussion}

We apply PCA to the data after pretreatment, and calculate the first principal component eigenvalue and eigenvector. Figure 7 shows the daily variation in the first principal component eigenvalue $\lambda$. The black arrows denote the days of the earthquake occurrence. We calculate the average and the standard deviation $\sigma$ using all of the values to recognize anomalous $\lambda$ values. Those anomalous values are then defined as values that exceed the average by more than $1 \sigma$.

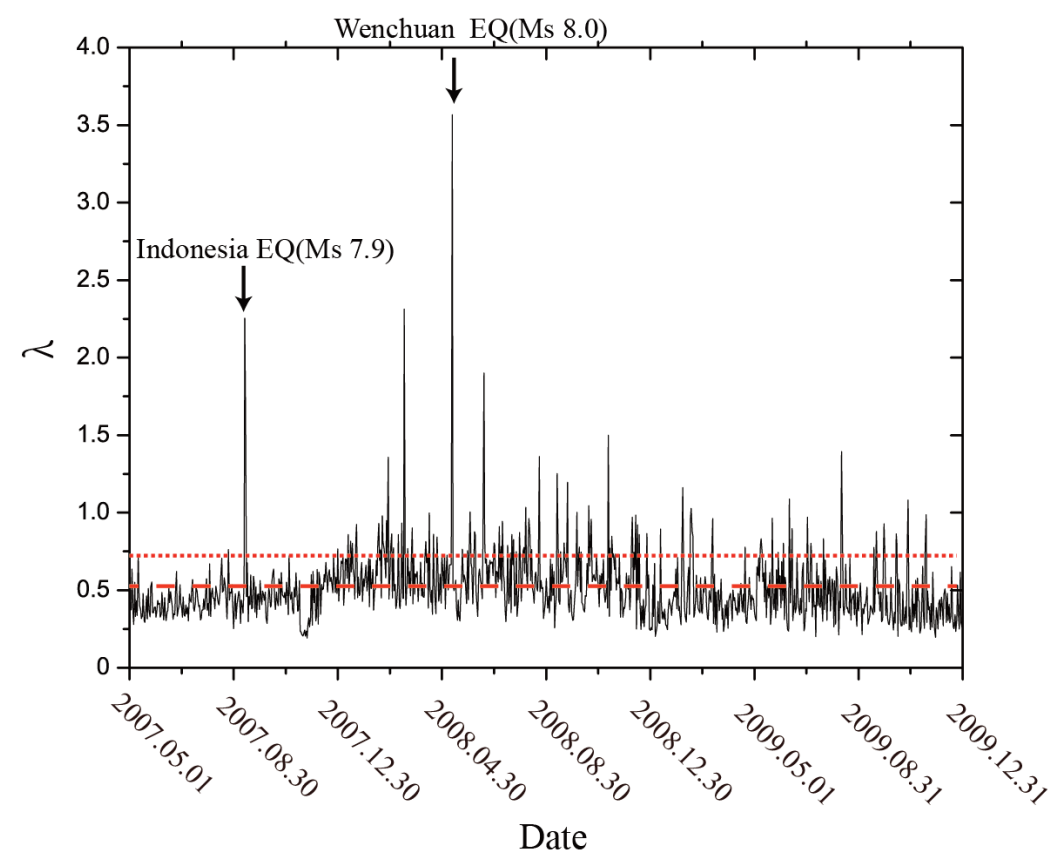

Figure 7. The results of the daily variations in the first principal component eigenvalue. The average value is delimited by a red horizontal dashed line, and the average of more than the standard deviation $\sigma$ is delimited by a red horizontal dotted line. The Indonesian earthquake occurred on September 12, 2007. The Wenchuan earthquake occurred on May 12, 2008.

The variations in $\lambda$ that are shown in Figure 7 illustrate that there are few anomalous values before January 2008 , and the anomalous value on September 12, 2007 was caused by the Indonesia earthquake. After January 2008, the number of anomalous eigenvalues began to increase, and this growth continued until a few months after the occurrence of earthquake.

In order to express the variation characteristics of the number of anomalous eigenvalues more intuitively, we calculate the cumulative number of anomaly eigenvalue and fit it by the sigmoidal function. Figure 8 illustrates the temporal behavior of $\mathrm{N}(\mathrm{t})$, denoted here as $\mathrm{N}$ (eigenvalue anomalies). It shows that a sigmoidal temporal behavior before the earthquake with a lower concavity and a subsequent sigmoidal behavior after, with an opposite concavity. We indeed notice an acceleration that occurs approximately 4 months before the earthquake (from January 2008), and a short period of earthquake quiescence that occurs before the earthquake. This process can be linked to the preparation phase of a large earthquake [Kei.,2011]. After such a large-magnitude earthquake, 


\section{Kaiguang Zhu et al.}

rearrangement of stresses in the crust commonly leads to subsequent occurrences of a large number of anomaly events [McCloskey et al.,2005; Parsons et al.,2002]. As is shown in Figure 8, there was a steep rise in the number of anomalous eigenvalues after the earthquake.

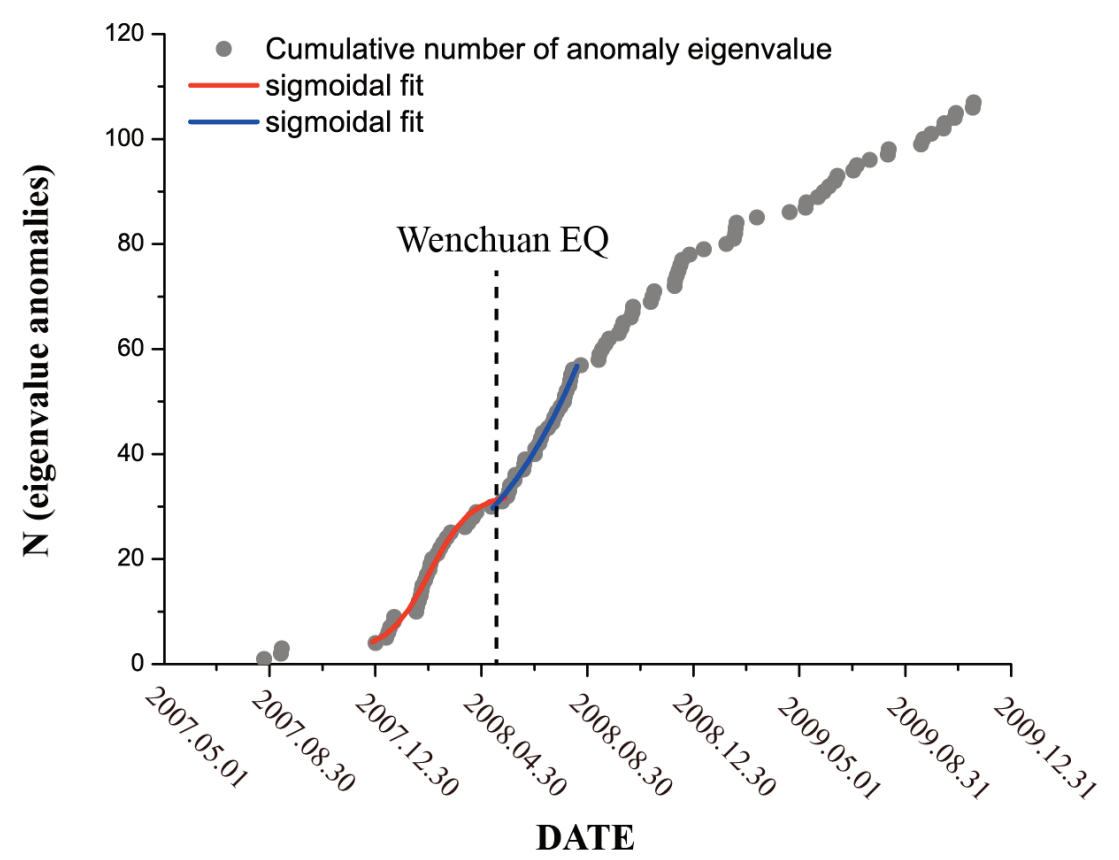

Figure 8. Cumulative number of anomalous eigenvalue calculated by the results of the daily variations in the first principal component eigenvalue. The earthquake day is represented as a vertical dotted line. The red and blue curves are the sigmoidal fits before and after the occurrence of the earthquake.

We consider that the eigenvalue and eigenvector are both obtained from the decomposition of the co-variance matrix of the data and that both contain parts of information of the data. We perform a combined eigenvalue and eigenvector analysis. The $\mathrm{x}$-axis and $\mathrm{y}$-axis respectively represent the $\theta$ and $\phi$ angles, and the color indicates the changes in eigenvalue, as is shown in Figure 9.

A boxplot is used to express the change of eigenvector before Wenchuan earthquake, as is shown in Figure 10, Figure 10(a) shows that the range of $\phi$ is less than $240^{\circ}$ (scope of black dotted lines), and it is mainly concentrated from $60^{\circ}$ to $120^{\circ}$. Between May 2007 and June 2007(scope of blue box), it shows a stable trend. Beginning in July 2007, the range of $\phi$ is almost $0^{\circ}$ to $360^{\circ}$, and this phenomenon makes eigenvectors present a trend of vertical diffusion in Figure 9. The boxplot of $\theta$ is shown in Figure 10(b), it also shows a stable trend between May 2007 and June 2007. Beginning in July 2007, the magnitude of the $\theta$ centralization range gradually increased, and this phenomenon makes eigenvectors present the trend of steady transverse diffusion shown in Figure 9.

MA Jin et al., [2014] performed a modeling study of instability on a planar strike-slip fault in the laboratory. They indicated that the occurrence of earthquakes is related to the synergism process of a fault, and the synergism process of a fault includes three stages. The first stage occurs when the stress curve deviates from linearity. In the second stage, the isolated areas of strain release increase and extend steadily. The third stage is the sections of strain release on the fault accelerate to expand and strain levels of strain-accumulation areas accelerate to rise. Wang et al., [2018] used the method of Cataclastic Analysis based on multiple focal mechanisms to study stress tensor evolution before the Wenchuan earthquake. The results show that the apparent stress change occurred in June 2007, and the stress increment distribution over the two periods before and after June 2007 indicates that the stress level is significantly weakened after June 2007.

The spatial distribution of eigenvectors and abnormal variation of eigenvalues clearly shows a consistent result. In this paper, the dimension of our principal component analysis is three, so the eigenvectors obtained have the same dimension in the vector space, and the tip of the vector moves on a sphere. 
Strain variation of the Wenchuan earthquake
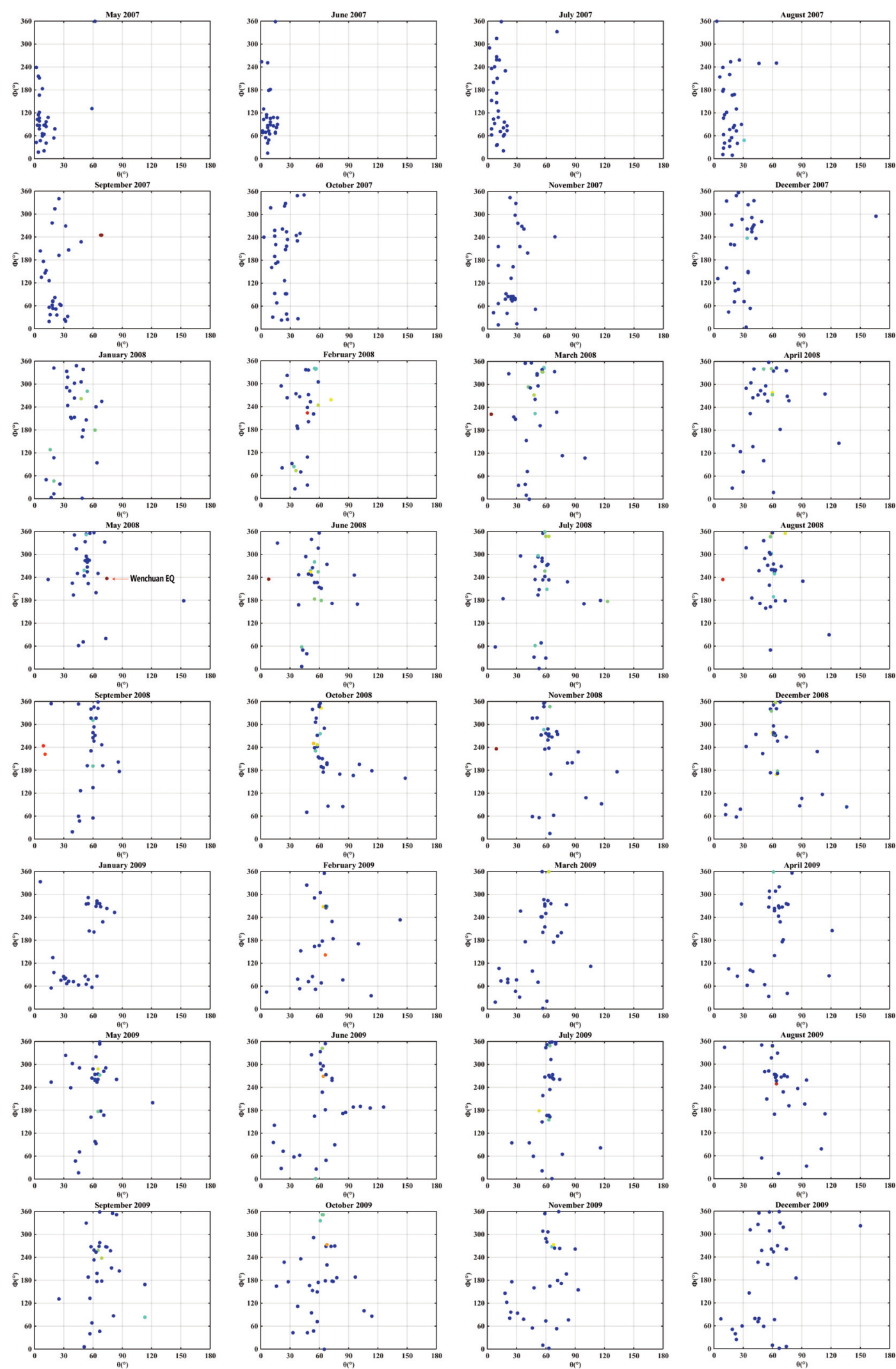

Figure 9. The result of the spatial distribution of eigenvectors and abnormal variation of eigenvalues. The $\mathrm{x}$-axis and $y$-axis respectively represent the $\theta$ and $\phi$ angles, and the color indicates the changes in eigenvalue. The red arrow denotes the day of the Wenchuan earthquake. The first stage is from May 2007 to June 2007. The second stage is from July 2007 to December 2007. The third stage from January 2008 to the occurrence of the earthquake. 


\section{Kaiguang Zhu et al.}
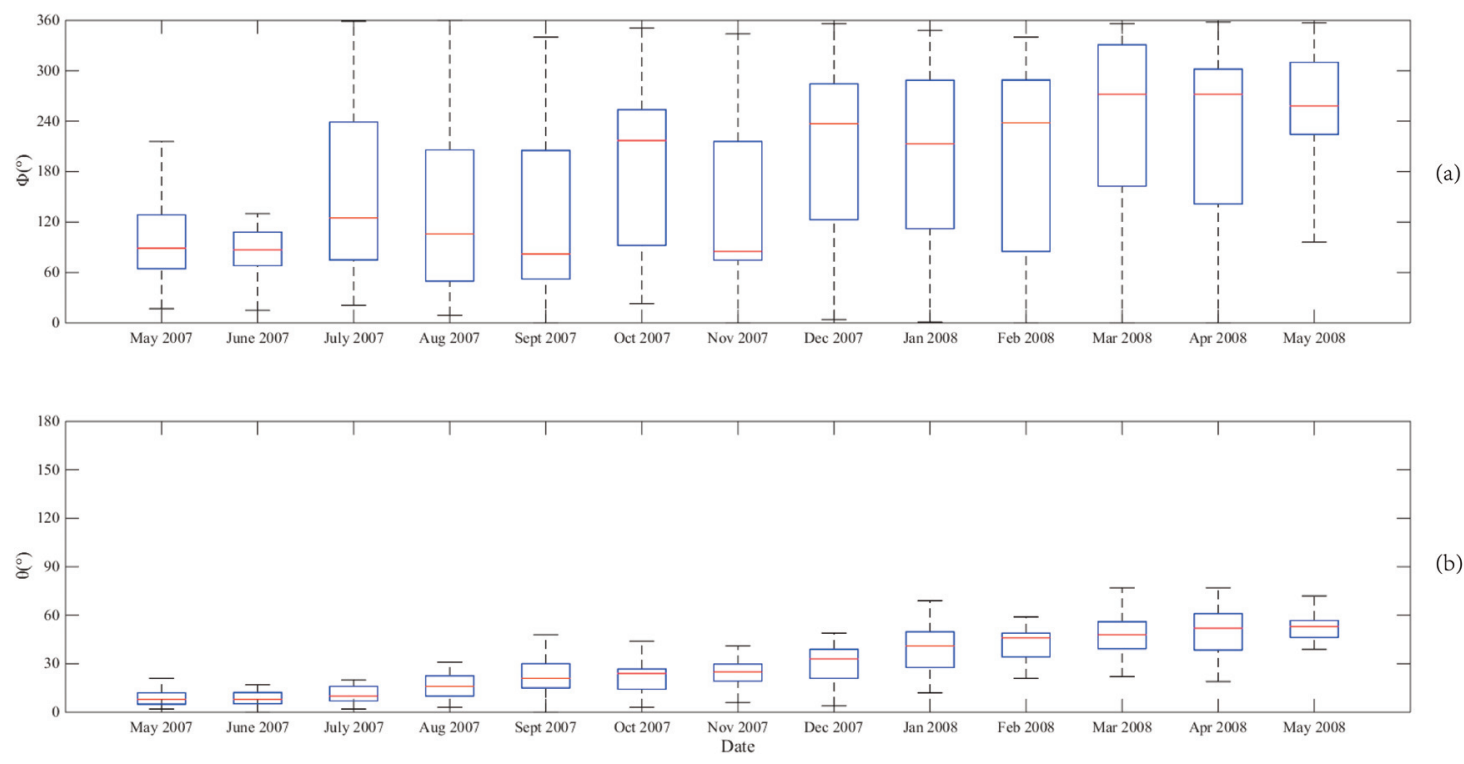

Figure 10. The result of the boxplot display for eigenvector angle per month from May 2007 to May 2008. (a) is a boxplot display of $\phi ;$ (b) is a boxplot display of $\theta$.

The $\theta$ and $\phi$ angles can uniquely represent an eigenvector, and they vary with eigenvectors. As it is shown in Figure 9 and Figure 10, between May 2007 and June 2007, the variations of $\theta$ and $\phi$ are very stable, showing a trend of vertical diffusion. This suggests that the spatial distribution of eigenvectors presents the steady state, and the beginning of divergence of strain variations of different portions indicates the first stage. The second stage begins in July 2007, when the $\theta$ and $\phi$ angles suggest steady transverse diffusion. Due to the strain, the isolated areas of strain release extend steadily, and the spatial distribution of eigenvectors suggests steady diffusion. When the strain levels of the strain-accumulation areas accelerate to rise, the number of anomaly eigenvalues begins to increase (from January 2008 onward), which signs the beginning of the third stage.

Based upon the results from the combined eigenvalue and eigenvector analysis, the spatial distribution of eigenvectors and accelerated occurrence of eigenvalue anomalies represent the stress evolution characteristics of the fault from the steady state to the sub-instability state in rock experiments [M.A. Jin et al., 2014]. We infer that this process may also be linked to the preparation phase of a large earthquake.

\section{Conclusions}

We have utilized the PCA technique to study the anomaly characteristics of borehole strain data that preceded the Wenchuan earthquake. The eigenvalues and eigenvectors of the first principal component of time series borehole strain data are used to analyze the characteristics of strain variation before the Wenchuan earthquake. The results of the analysis indicate that the borehole strainmeter of Guza station recorded the preparation phase of the Wenchuan earthquake, and PCA is capable of effectively extracting the features of crustal strain changes. The principle of crustal movement is complex. In the application of PCA to investigate strain earthquake precursors, it is difficult to determine the physical meaning of eigenvalues and eigenvectors. In future work, we aim to confirm the physical meaning of eigenvalues and eigenvectors. By applying PCA to describe the strain behavior, we may be able to ensure that the PCA technique has great potential in the study of earthquake precursors.

Acknowledgements. The authors are grateful to the Key Laboratory of the Geo-Exploration Instrumentation of Ministry of Education in Jilin University of China. This research was supported by the National Natural Science Foundation of China under Grant No.41974084. 


\section{References}

Asai Y., M. Okubo, H. Ishii, H. Aoki, T. Yamauchi, Y. Kitagawa, Naoji Koizumi (2005). Co-seismic strain-steps associated with the 2004 off the Kii peninsula earthquakes-Observed with Ishii-type borehole strainmeters and quartz-tube extensometers, Earth, planets and space, 57(4), 309-314.

Barbour A.J and D. C. Agnew (2012). Detection of seismic signals using seismometers and strainmeters, Bull. Seismol. Soc. Am. 102(6), 2484-2490.

Chi S L., Y. Chi, T. Deng, C. W. Liao, X. L. Tang and L. Chi (2009), The Necessity of Building National StrainObservation Network from the Strain Abnormality Before Wenchuan Earthquake, Recent Developments In World Seismology (in Chinese), 1, 1-13.

Chun Chieh Hsiao, J .Y. Liu , K.I. Oyama (2010). Seismo-ionospheric precursor of the 2008 Mw7.9 Wenchuan earthquake observed by FORMOSAT-3/COSMIC, GPS Solut. 14:83-89, doi: 10.1007/s10291-009-0129-0.

Fu G.Y., X.Z. Shen., Y. Fukuda, S. H. Gao and S. Yoshii (2011). Co-seismic strain changes of Wenchuan Mw7.9 earthquake recorded by borehole strainmeters on MTibetan plateau, Geod. Geodyn., 2(3):42-49, doi:10.3724/SP.J.1246.2011.00042.

Gladwin M. (1984). High-precision multi-component borehole deformation monitoring. Rev. Scientific Instrums. 55(12): 2011-2016.

Gómez Londoño E., L. Castillo López, K. Thaís de Souza (2005). Using the Karhunen-Loeve transform to suppress ground roll in seismic data. Earth Sci. Res. J., 9 (2): 139-147.

Hattori K., A. Serita, K. Gotoh, C. Yoshino, M. Harada, N. Isezaki, M. Hayakawa (2004). ULF geomagnetic anomaly associated with 2000 Izu islands earthquake swarm, Japan. Phys. Chem. Earth, Parts A/B/C. 29(4): 425-435.

Hautmann. S., D. Hidayat., N. Fournier., A. T. Linde., I. S. Sacks. and C. P. Williams (2013). Pressure changes in the magmatic system during the December 2008/January 2009 extrusion event at Soufriere Hills Volcano, Montserrat (W.I.), derived from strain data analysis, J. Volcanol. Geotherm. Res. 250, 34-41.

Lin J.W (2015). Early warning of tsunami from seismo-ionospheric fluctuation after Japan's March 11, 2011, M=9.0 Tohoku earthquake using two-dimensional principal component analysis, Ann. Geophys., 58, 4, A0442, doi:10.4401/ag-6710.

Kei Katsumata (2011). Precursory seismic quiescence before the Mw=8.3 Tokachi-oki, Japan, earthquake on 26 September 2003 revealed by are examined earthquake catalog, J. Geophys. Res., 116 (B10307), doi:10.1029/2010JB007964.

Linde, A. T., M. T. Gladwin., M. J. S. Johnston., R. L. Gwyther and R. G. Bilham (1996). A slow earthquake sequence on the San Andreas fault, Nature, 383.65-68.

Liu C. C., A.T. Linde, I.S. Sacks (2009). Slow earthquakes triggered by typhoons, Nature, 459, (7248): 833-836.

Liu Q, J. Zhang (2011). Application of S Transform in Analysis of Strain Changes Before and After Wenchuan Earthquake, J. Geod. Geodyn. 31(4). 6-9.

McGarr A., I.S. Sacks, A.T. Linde, S. M. Spottiswoode, R. W. E. Green (1982). Coseismic and other short-term strain changes recorded with Sacks-Evertson strainmeters in a deep mine, South Africa, Geophys. J. Int., 70(3): 717740.

McCloskey, J., S. S. Nalbant, and S. Steacy (2005). Indonesian earthquake: Earthquake risk from co-seismic stress, Nature, 434, 291.

MA J, Y. S. Guo (2014). Accelerated Synergism Prior to Fault Instability: Evidence From Laboratory Experiments and an Earthquake Case, Seismol. Geol. (In Chinese), 36(3), doi:10.3969/j.issn.0253-4967.2014.03.001.

Ouyang, Z.X. (1977). RDB-1 type electric capacity strainmeter, Selected papers of the national conference on stress measurement, Part. 2 (in Chinese). 337-348.

Parsons, T. (2002). Global Omori law decay of triggered earthquakes: Large aftershocks outside the classical aftershock zone, J. Geophy. Res. 107, doi:10.1029/2001JB000646.

Qiu Z.H., B. H. Zhang, S. L. Chi, L. Tang and M. Song (2011). Abnormal strain changes observed at Guza before the Wenchuan earthquake. Science China Earth Sciences, 54(2): 233-240, doi: 10.1007/s11430-010-4057-1.

Qiu Z.H, B. H. Zhang (2002). Current situation of the seismological networks for borehole stress-strain precursor surveillance in China. Recent Developments in World Seismology (in Chinese), 6: 5 9.

Qiu Z.H., Y. Shi (2003). Observations of remote coseismic stress step-changes. Science in China Series D: Earth Sciences. 46: 75-81. 


\section{Kaiguang Zhu et al.}

Qiu Z.H., Y. Shi (2004). Application of observed strain steps to the study of remote earthquake stress triggering, Earthquake Science, 17(5): 534.

Qiu Z.H., B. H. Zhang, Chi S.L., L. Tang, M. Song (2011). Abnormal strain changes observed at Guza before the Wenchuan earthquake. Science China Earth Sciences. 54(2): 233-240.

Qiu Z.H., L. Tang, B. Zhang, Y. Guo (2013). In situ calibration o, f and algorithm for strain monitoring using fourgauge borehole strainmeters (FGBS), J. Geophys. Res,: Solid Earth, 118(4): 1609-1618.

Qiu Z.H., S. Chi S., Z. Wang, S. Carpenter, L. Tang, Y. Guo, G. Yang (2015). The strain seismograms of P-and S-waves of a local event recorded by four-gauge borehole strainmeter, Earthquake Science, 28(3): 209-214.

Sacks I.S, D. W. Evertson (1971). Sensitive borehole strain-rate meter [J]. Carnegie Inst, Washington Yearb, 68: 448 453 .

Sacks, I. S., S. Suyehiro., A. T. Linde and J. A. Snoke (1978). Slow earthquakes and stress redistribution, Nature. 275, 599-602.

Sacks, I. S., A. T. Linde, J. A. Snoke and S. Suyehiro (1981). A Slow Earthquake Sequence Following the Izu-Oshima Earthquake of 1978, Earthquake Prediction, 617-628.

Wu Z.J, H. J. Wu, F. Han (2017). Two-Dimension Elasticity, in Elasticity, $2^{\text {nd }}$ ed., Beijing, China, 5, 95-98.

Sturkell, E., K.Agustsson, A.T.Linde, S.I.Sacks, P.Einarsson, F.Sigmundsson, H.Geirsson, R.Pedersen, P.C.LaFemina, and H.Olafsson (2013). New insights into volcanic activity from strain and other deformation data for the Hekla 2000 eruption. J. Volcanol. Geotherm. Res. 256, 78-86.

Telesca L., G. Colangelo, K. Hattori and V. Lapenna (2004). Principal component analysis of geo-electrical signals measured in the seismically active area of Basilicata Region (southern Italy). Nat. Hazards Earth Syst. Sci. 4, (5/6): 663-667.

Voight, B., A.T. Linde, I.S. Sacks, G. S. Mattioli, R.S.J. Sparks, D. Elsworth, D. Hidayat, P. E. Malin, E. Shalev, C. Widiwijayanti, S. R. Young, V. Bass, A. Clarke, P. Dunkley, W. Johnston, N. McWhorter, J. Neuberg, P. Williams (2006). Unprecedented pressure increase in deep magma reservoir triggered by lava-dome collapse. J. Geophys. Res. Lett. 33, L03312, doi:10.1029/2005GL024870.

Wang K.Y, Y. S. Guo, X. D. Feng (2018). Sub-instability stress state prior to the 2008 Wenchuan earthquake from temporal and spatial stress evolution. Chinese J. Geophys. (in Chinese). 61(5):1883-1890, doi:10.6038/cjg2018M0225. 